\title{
Does Scientific Progress Consist in Increasing Knowledge or Understanding?
}

\begin{abstract}
Bird (2007) argues that scientific progress consists in increasing knowledge. Dellsén (2016a) objects that increasing knowledge is neither necessary nor sufficient for scientific progress, and argues that scientific progress rather consists in increasing understanding. Dellsén also contends that unlike Bird's view, his view can account for the scientific practices of using idealizations and of choosing simple theories over complex ones. I argue that Dellsén's criticisms against Bird's view fail, and that increasing understanding cannot account for scientific progress, if acceptance, as opposed to belief, is required for scientific understanding.
\end{abstract}

\section{Keywords}

Epistemic Approach, Knowledge, Noetic Approach, Scientific Progress, Understanding

Park, Seungbae (2017). "Does Scientific Progress Consist in Increasing Knowledge or Understanding?”, Journal for General Philosophy of Science 48 (4): 569-579.

Acknowledgement: This paper improved a lot thanks to the insightful comments from anonymous referees and Dr. Beisbart.

Seungbae Park

Ulsan National Institute of Science and Technology

Republic of Korea

nature@unist.ac.kr

\section{Introduction}

Alexander Bird (2007) defends the epistemic approach to scientific progress, according to which scientific progress consists in increasing knowledge. Finnur Dellsén (2016a) proposes an original alternative approach to scientific progress which he calls the noetic approach. ${ }^{1}$ According to the noetic approach, scientific progress consists in increasing understanding. He argues that the noetic approach is superior to the epistemic approach. This paper aims to defend the epistemic approach from Dellsén's criticisms ${ }^{2}$ and to criticize the noetic approach. There are other approaches to scientific progress in the literature. They are the semantic approach (Niiniluoto, 1980; 2014) and the problem-solving approach (Kuhn, 1962/1970; Laudan, 1977; 1984), but these approaches fall outside the scope of this paper.

The outline of this paper is as follows. In Section 2, I spell out the epistemic and noetic approaches, and then reply to referees' objections to the epistemic approach. In Section 3, I attempt to refute Dellsén's contention that there can be scientific progress and an increase in understanding, even without any accumulation of knowledge. In Section 4, I attempt to confute his contention that even if there is an accumulation of knowledge, there can be no scientific progress and no increase in understanding. In Sections 5 and 6, I reply to his objections that the noetic approach accounts for, while the epistemic approach cannot, the scientific practices of using idealizations and of choosing simple theories over complex ones.

\footnotetext{
1 'Noetic' comes from the Greek 'nous' which means understanding (Dellsén, 2016: 72, footnote 2).

2 See Darrell Rowbottom (2010), Ilkka Niiniluoto (2015), and Juha Saatsi (2016) for other criticisms against the epistemic approach.
} 


\section{Epistemic and Noetic Approaches}

Bird says that "an episode in science is progressive when at the end of the episode there is more knowledge than at the beginning" (2007: 64). He also says that "progress is made when certain means to an end are achieved" (2007: 83). So the epistemic approach comes down to the suggestion that scientific progress consists in increasing knowledge or in achieving a means to increase knowledge. Let me call the second part of the epistemic approach the means-end thesis. It implies that an episode is progressive, even if there is no more knowledge at the end of it than there was at the beginning of it, insofar as it paves the way for the production of knowledge in subsequent research. Suppose for the sake of argument that the history of science was filled with discarded theories, that past scientists did not generate knowledge, including observational knowledge, and that they kept researching despite repeated failures. Even so, they made progress, as long as their practices will contribute to the generation of knowledge in subsequent researches. ${ }^{3}$

How would critics respond to the means-end thesis? They might object that the thesis is too permissive (referee). Not everything that promotes the accumulation of scientific knowledge counts as scientific progress. For example, technological advances and increased funding for scientific research promote the growth of scientific knowledge, but hardly count as scientific progress in the relevant sense. It is not clear on what grounds the means-end thesis excludes those developments that promote knowledge but do not count as scientific progress.

My response to this objection is to point out that the debate over scientific progress is restricted to those episodes that involve cognitive changes. Increased funding is not an episode that involves a cognitive change. Unlike increased funding, confirming a hypothesis is an episode that involves a cognitive change. So increased funding might be called a noncognitive episode; confirming a hypothesis might be called a cognitive episode. Participants in the debate over scientific progress are in the business of determining whether a cognitive episode is progressive or not, not whether a non-cognitive episode is progressive or not. In light of this observation, let me formulate the epistemic approach more precisely as the view that a cognitive episode in science is progressive, if and only if it involves the increase in knowledge or the acquisition of a means to increase knowledge.

Critics might also object that the means-end thesis makes it difficult to discriminate, within the whole of the activities that are referred to as 'scientific inquiry,' the episodes that count as instances of cognitive progress (another referee). Indeed, in light of the means-end thesis, far too many things seem to count as progress. Epistemists would view the mere fact that scientists keep researching despite repeated mistakes as progressive. Given how broad the notion of progress becomes in view of the means-end thesis, it seems legitimate to ask what part or phase of scientific inquiry would not count as progress on the epistemic account.

My response to this worry is to admit that there can be an epistemological problem over whether a cognitive episode does or does not serve as a means to obtain knowledge. This epistemological problem, however, does not denigrate the means-end thesis. To use an analogy, the correspondence theory of truth holds that a statement is true if and only if it corresponds to a state of affairs. There can be an epistemological problem over whether a particular statement corresponds to a state of affairs or not. This epistemological problem,

\footnotetext{
${ }^{3}$ Bird conjectures, a referee points out, that "the relevant developments that promote knowledge will themselves be knowledge" $(2007,84)$. In my view, however, Bird seems to mean gathering observational knowledge by 'the relevant developments.' In any event, the epistemic approach requires only the mean-end thesis.
} 
however, does not denigrate the correspondence theory of truth. It is one thing to propose a criterion to distinguish between true and false statements; it is another whether we have enough evidence or not to tell whether a particular statement is true or false. Similarly, it is one thing to propose a criterion to distinguish between progressive and non-progressive episodes; it is another whether we have enough evidence or not to tell whether a particular episode is progressive or non-progressive. The referee's objection, however, shows that when epistemists claim that a cognitive episode is progressive, they have the burden to show that there is an increase in knowledge in the episode, or that the episode serves as a stepping stone for the growth of knowledge in subsequent research.

Let me turn to Dellsén's conception of scientific understanding. He says that an "agent has partial scientific understanding of a given target just in case she grasps how to correctly explain and/or predict some aspects of the target in the right sort of circumstances" (2016a: 75). An explanation is "correct even though it appeals to theories that are strictly speaking false, e.g. idealizations and simplifications, provided that those theories help identify the explanatorily relevant factors on which the explanandum depends" (Dellsén, 2016a: 75). In addition, "the explanatorily/predictively essential elements of a theory must be true in order for the theory to provide grounds for understanding" (Dellsén, 2016a: 73, footnote).

The foregoing conception of scientific understanding is built into the noetic approach, according to which "scientific progress consists in increasing understanding," and "an episode in science is progressive precisely when scientists grasp how to correctly explain or predict more aspects of the world at the end of the episode than at the beginning" (Dellsén, 2016a: 73). The noetic approach does not embed the means-thesis (referee). Dellsén states explicitly that "since nearly anything can promote progress, we must be careful not to confuse scientific progress itself with the promotion of such progress" (2016a: 73). So he would not say that an episode is progressive on the grounds that a means to increase understanding is gained in the episode.

Let me apply the noetic approach to the series of the three theories of diseases in the history of science. The humoral theory, the miasma theory, and the germ theory hold, respectively, that a disease results from an imbalance of the four humors, from a noxious air, and from germs. If the germ theory is true, the humoral theory and the miasma theory failed to identify the explanatorily relevant factors. The noetic approach, however, implies that there was scientific progress in the transition from the humoral theory to the miasma theory, provided that the miasma theory predicted more phenomena than the humoral theory. According to the noetic approach, scientific progress requires not both increasing explanations and predictions, but increasing explanations or predictions.

\section{Is Knowledge Necessary for Scientific Progress?}

Philip Kitcher (2002), Peter Lipton (2004), and Stephen Grimm (2006, 2014) claim that belief and justification are essential components of understanding. Dellsén, however, rejects their view, arguing that understanding requires neither belief nor justification, and that in certain cases in science, there is no increase in knowledge, but there is an increase in understanding, and the increase in understanding matches scientific progress. In other words, what is necessary for scientific progress is not increasing knowledge but increasing understanding. I flesh out and criticize Dellsén's argument for this contention in this section.

Dellsén argues that belief is not required for understanding, and that scientific progress consists in increasing understanding, so increasing knowledge is not necessary for scientific progress. So we need to examine how he argues that understanding does not require belief. He (2016b: 10) appeals to L. Jonathan Cohen's (1992) distinction between belief and 
acceptance. For Cohen, to believe $\mathrm{p}$ is to feel it to be true, and to accept $\mathrm{p}$ is to adopt the policy of including $\mathrm{p}$ "among one's premises for deciding what to do or think in a particular context" (1992: 4). To use Cohen's example, a lawyer may accept that her client is innocent in court. Once she accepts the proposition, she can infer that the prosecutor's witness is not reliable, and can speak to the judge accordingly. Just as the lawyer merely accepts the proposition, so a physicist may merely accept string theory, and then use it "to explain any fundamental physical phenomena just as well as someone who also believes string theory" (Dellsén, 2016b: 11). It is not required that agents believe that theories are true - they "may merely assume or accept that they are true for the purposes of explanation and or prediction" (Dellsén, 2016a: 76). In short, "understanding something may merely involve treating certain propositions or theories as given in the context of explaining something, as opposed to being disposed to feel that the propositions or theories are true" (Dellsén, 2016b: 10).

Contrary to what Dellsén claims, however, scientific understanding does require belief. In science, a tragic result may occur, if understanding lacks belief. Imagine that you are a scientist, and that you tested the hypothesis that the low intake of folic acid during pregnancy increases the chance of having premature babies. You studied one hundred pregnant women. The half and the other half of them took, respectively, less than two hundred micrograms and more than three hundred micrograms of folic acid a day during pregnancy. It turned out that twenty women of the first group and four women of the second group gave birth to premature babies. Now, you submit your research paper to a scientific journal. The editors ask, "Are the one hundred pregnant women in your study real or imaginary?" You answer, "I don't know whether they are real or imaginary. I don't believe they are real. But I accept that they are real. If we adopt the policy of using that assumption for the purpose of explanation, we can understand why the twenty women had premature babies." The editors will be perplexed by your answer. They have two possible responses to you. One is kind; the other is unkind. Their kind response is to let you know that science is an inquiry into the world, but that they suspect that your manuscript is not an outcome of an inquiry into the world. Their unkind response is to accuse you of having fabricated data, a serious violation of research ethics in science. Of course, you did not fabricate the data, and the data are true. But the point is that if you do not believe that they are true, you are opening yourself to the charge of the serious misconduct, and to the charge that your understanding does not reflect the world. Belief plays such a crucial role in science.

When scientists explain something in terms of a scientific theory, they believe that it is real. For example, when they invoke Newtonian mechanics to explain why the Earth turns around the Sun, they believe that the Earth turns around the Sun. ${ }^{4}$ If they do not believe that something is real, they do not bother to explain it. It is for this reason that biologists do not bother to explain why a unicorn has a horn, and that Ptolemaic astronomers did not bother to explain why the Earth revolves around the Sun. If scientists explain something that they do not believe to be real, they face a disconcerting question: "What is the point of explaining something you do not believe to be real?" Thus, it is in part because scientists believe that their explananda are real that their peers take their explanations seriously.

How about predictions? Suppose that you are a scientist and use Newtonian mechanics to send a rocket to the Moon. You predict that the rocket will arrive on the Moon at a particular time. Before you confirm the prediction, you may merely accept it. Once you

\footnotetext{
${ }^{4}$ Should they believe that Newtonian mechanics is true? Dellsén would say no, but I (2015: 226-228; 2016 : 77 78; 2017: 59-60) argue that problems arise when we explain something in terms of a scientific theory without believing that it is (approximately) true. This paper, however, assumes for the sake of argument that Dellsén is right, i.e., that it is legitimate merely to accept a scientific theory and then use it to explain phenomena.
} 
confirm it, however, you believe it. If you still merely accept it, you can be accused of making no discrimination between confirmed and unconfirmed predictions, and more importantly, your peers might dismiss your prediction, saying, "Why should I believe what you don't?" They may even suspect that you have fabricated data. Thus, it is in part because scientists believe that their predictions are true that their peers take their predictions seriously.

To summarize, scientists believe at least that explained phenomena are real, and that their confirmed predictions are true. In the absence of such beliefs, their peers might suspect that their explanations and predictions do not concern the world, and that their understandings, which allegedly arise from their explanations and predictions, do not reflect the world. Thus, it is not acceptance but belief that is required for scientific understanding.

Let me now turn to Dellsén's contention that understanding does not require justification. The idea is that understanding results from explanations and predictions making use of propositions, and that understanding can arise without any justification for the propositions. He states this view as follows:

..there is no requirement in $(\mathrm{U})$ that an agent has epistemic justification for the propositions on which her understanding is based. More precisely, an agent may partially understand something by virtue of grasping how to correctly explain or predict some aspect of it, even though she does not have the epistemic justification required for knowing the propositions to which she appeals in her explanations/predictions. (2016a: 76)

In order to illustrate this point, Dellsén (2016a: 76) offers the example of Einstein's explanation of Brownian motion in terms of the kinetic theory. He cites Einstein's following paragraph:

In this paper it will be shown that according to the molecular-kinetic theory of heat, bodies of microscopically-visible size suspended in a liquid will perform movements of such magnitude that they can be easily observed in a microscope, on account of the molecular motions of heat. It is possible that the movements to be discussed here are identical with the so-called "Brownian molecular motion"; however, the information available to me regarding the latter is so lacking in precision, that I can form no judgment in the matter. (Einstein, 1905/1956: 1)

Einstein did not have enough information to determine whether the movements under investigation were Brownian motion, so "the explanandum in Einstein's explanation of Brownian motion did not constitute knowledge for Einstein at the time" (Dellsén, 2016a: 76). In addition, "the kinetic theory of heat was very much up for debate at the turn of the $20^{\text {th }}$ century, with many physicists favoring alternative theories of heat that did not assume the existence of submicroscopic molecules" (Dellsén, 2016a: 76). In short, Einstein was neither justified in believing that the explanandum was true, nor justified in believing that the explanans was true. Yet, he explained Brownian motion in terms of the kinetic theory, thereby giving rise to the understanding of Brownian motion and making scientific progress. Therefore, there can be an advance in scientific understanding and progress without justification.

Does this episode spell doom for the epistemic approach? Epistemists would argue that Einstein believed that a certain phenomenon existed although he was not sure whether it was Brownian motion or not. He did not call the phenomenon Brownian motion because the name 'Brownian motion' was a theory-laden term presupposing the existence of microscopic particles. Moreover, he made scientific progress simply because his insight into the relationship between the kinetic theory and Brownian motion served as a means to establish 
the existence of molecules in the subsequent research by Jean Perrin in 1908. Thus, the aforementioned means-end thesis can be utilized to defend the epistemic approach from Dellsén's objection.

Let me now turn to another example from Dellsén (2016a: 80). It involves Alfred Wegener's continental drift theory. Intuitively speaking, Wegener made scientific progress when he advanced the continental drift theory in the early twentieth century. The epistemic approach, however, incorrectly implies that he did not on the grounds that his theory was not supported by sufficient evidence. By contrast, the noetic approach correctly implies that he did on the grounds that his theory correctly explained the similarity between the Western coastline of Africa and the Eastern coastline of South America.

How would epistemists respond to this episode? They would say that Wegener's contribution paved the way for the advent of the theory of plate tectonics in the 1960s. The two theories share the key assumption that continents move around. The main difference between them is that only the theory of plate tectonics specifies how continents, massive things, move around. Thus, the means-end thesis can also be utilized to defend the epistemic approach from Dellsén's objection involving Wegener's continental drift theory.

The foregoing examples of Einstein and Wegener illustrate that understanding sometimes acts as a vehicle to arrive at knowledge. Einstein showed how the kinetic theory was related to Brownian motion, and Wegener showed how the continental drift theory was related to the similarity between the two coastlines. They showed how some propositions are related to one another, thereby making preparations for the arrival of knowledge in the subsequent researches.

Finally, let me elucidate a noteworthy implication of Dellsén's stripping understanding of justification. This impoverished notion of understanding implies that understanding does not entitle us to make any inference about the world. To use the Einstein example, suppose that Einstein understood Brownian motion in terms of the kinetic theory, and that he was not justified in believing that the kinetic theory was true, and that Brownian motion was real. Under these conditions, he would not have been entitled to infer, for example, that water is composed of molecules. Thus, if understanding is devoid of justification, we are only entitled to infer some propositions from what we understand, without having any idea whether they correctly reflect the world or not.

\section{Is Knowledge Sufficient for Scientific Progress?}

Dellsén contends that in science there are cases in which there is an increase in knowledge, but there is no increase in understanding and no scientific progress. In other words, what is sufficient for scientific progress is not increasing knowledge but increasing understanding. I spell out and criticize his argument for this contention in this section.

Dellsén says that "knowledge is not sufficient for understanding because understanding involves a holistic cognitive state that goes beyond having knowledge of individual propositions" (2016a: 76). For example, "I may know the length of a flagpole's shadow, the flagpole's height, the position of the sun, and the rectilinear propagation of light, and yet fail to grasp how to explain or predict the length of the flagpole's shadow" (2016a: 75). In other words, I may know that an explanandum and explanantia are true, but not understand the explanandum in terms of the explanantia.

This example, however, does not show that knowledge is not sufficient for understanding. You do not understand why the shadow is of a certain length simply because you do not have the inferential knowledge necessary to see how the length of the shadow is related to the length of the flagpole, the position of the sun, and the rectilinear propagation of 
light. Once you know how the explanandum follows from the explanantia, you will acquire the understanding of the explanandum in terms of the explanantia. In short, if you have all the relevant knowledge, you will have the relevant understanding.

Noetists would object that even if it is true that understanding requires inferential knowledge, it does not show that it is impossible to have knowledge without understanding (referee). Knowledge of the individual facts does not constitute understanding, even if inferential knowledge is additionally required for understanding. The claim that we can have knowledge without understanding seems all that Dellsén needs. The crucial question for scientific progress is whether acquiring knowledge of the individual explanantia and the explanandum without acquiring the inferential knowledge of how the explanandum follows from the explanantia always constitutes scientific progress.

It would be interesting to see what example noetists can come up with to show that even if there is all the relevant knowledge, there can be no relevant understanding. In addition, epistemists would distinguish between significant and insignificant knowledge. The knowledge of individual facts might be relatively insignificant, but the combination of the knowledge with the inferential knowledge might be relatively significant. The epistemic approach stands, even if the acquisition of insignificant knowledge does not constitute scientific progress, or constitutes insignificant scientific progress. This point will become clearer in the course of examining Dellsén's following objection to the epistemic approach.

Dellsén argues that "there are non-progressive episodes in which knowledge of theories or phenomena is accumulated while there is no increase in scientific understanding" (2016a: 77). In other words, there can be an accumulation of knowledge without any scientific progress or increase in understanding. Suppose that scientists gather data about childbirth rates and stork populations around Berlin, and that there is a statistical correlation between the two. Despite this correlation, our intuition says that "this is not a case of scientific progress" (2016a: 78). Gathering such data is not a progressive episode because the accumulation of such trivial knowledge is a symptom of degenerate science:

However, a scientific practice organized around accumulating trivial knowledge of this kind would seem to be a paradigm example of degenerate science. Note, for example, that no selfrespecting scientific journal would publish results of this kind, except as a parody. Similarly, research proposals outlining investigations of these sorts would not be taken seriously by any scientific funding agency. (Dellsén, 2016a: 79)

From the noetic point of view, it is not surprising why funding agencies and scientific journals would not be interested in the data about childbirth rates and stork populations. The correlation "is useless for understanding the natural world" (Dellsén, 2016a: 78). Thus, the noetic approach can explain, while the epistemic approach cannot, why there is no scientific progress in this case.

A similar criticism, however, can be leveled at the noetic approach. Suppose that you submit a research proposal to funding agencies. The proposal outlines explanations of why one million individuals have the numbers of hairs on their heads that they have. For example, Alice has 1,020 hairs on her head because she had 1,030 hairs, but lost 10 hairs while taking a shower. Bob has 1,000 hairs on his head because he had 1,001 hairs, but just pulled a gray one out. Once your research is completed, there will be one million such explanations. Your research will yield understandings of why one million individuals have the numbers of hairs that they have, but will impress neither funding agencies nor scientific journals.

Noetists would reply that we should distinguish between significant and insignificant understandings. Funding agencies and scientific journals are interested in significant 
understandings, but not in insignificant ones. So they are not interested in the understandings of why the one million individuals have the numbers of hairs that they have. On the noetic approach, however, scientific progress consists in increasing significant understanding, but not in increasing insignificant understanding. Increasing insignificant understanding constitutes no scientific progress or constitutes at best insignificant scientific progress. The noetic approach is refuted not when insignificant understanding does not make any difference to scientific progress but when significant understanding does not make any difference to scientific progress.

Epistemists, however, would say the same thing about Dellsén's objection that collecting the data about the childbirth rates and the stork populations does not count as scientific progress. We should distinguish between significant and insignificant knowledge. Funding agencies and scientific journals are interested in significant knowledge, but not in insignificant knowledge. So they are not interested in the data. On the epistemic approach, however, scientific progress consists in increasing significant knowledge, but not in increasing insignificant knowledge. Increasing insignificant knowledge constitutes no scientific progress or constitutes at best insignificant scientific progress. The epistemic approach is confuted not when insignificant knowledge does not make any difference to scientific progress but when significant knowledge does not make any difference to scientific progress.

\section{Idealizations}

Many scientific theories involve idealizations, i.e., they distort some factors and ignore others to make target systems tractable. Since those theories do not correctly represent their target systems, they are false. Regarding idealized theories, Dellsén raises two interesting objections against the epistemic approach. I reply to them in this section.

First, Dellsén says that "the epistemic account also (incorrectly) implies that minimalist idealizations ought to abate as science progresses, since there would be no point in having idealized theories from a purely epistemic point of view when non-idealized versions of these theories have been made available" (2016a: 81). Epistemists, however, would reply that a non-idealized theory might be useless to generate observational knowledge. We should consider the increase and the decrease in observational knowledge when we determine whether idealizations are beneficial or detrimental to the accumulation of knowledge. Thus, theoretical knowledge is not the only kind of knowledge that we should take into account in determining whether scientific progress is made.

Second, Dellsén says that "since idealized theories are not true, the epistemic account (incorrectly) implies that no progress is made when one generates minimalist idealizations, at least not when more accurate, non-idealized theories are available" (2016a: 81). Epistemists, however, would reply that the generation of idealized theories counts as progress, given that they facilitate inferences about observables, and that those inferences are accompanied by an accumulation of observational knowledge. In addition, they generate what I call approximate theoretical knowledge, given that they, although false, are approximately true. ${ }^{5}$ Generating approximate theoretical knowledge is better for scientific progress than not generating such knowledge at all.

Moreover, noetists need the notion of approximation as epistemists do. Suppose that scientists performed experiments and obtained data about a given target, and that the data

\footnotetext{
5 Approximate knowledge concerns an approximately true proposition. Suppose, for example, that I know that Alice is $170.1 \mathrm{~cm}$. Then, I approximately know that she is $170 \mathrm{~cm}$.
} 
slightly deviate from the expected data. Noetists would not say that scientists have made no progress on the grounds that the actual data only approximate the expected data. They would rather say that scientists made progress on the grounds that they understand the target more than before as a result of obtaining such imperfect data.

Noetists would reply that although they also need the notion of approximation, it may be easier for them to incorporate this notion into their account since according to them, understanding requires acceptance rather than belief (referee). The referee's idea seems to be that instead of believing that the actual data are approximately true, scientists can accept that they are true.

The referee's suggestion indicates that on the noetist account, scientists can merely accept not only scientific theories but also data. As I argued earlier, however, if scientists merely accept data, they are opening themselves to the charge that they have fabricated the data, and to the charge that their explanations, predictions, and understandings do not reflect the world. To merely accept data is to obfuscate the connection between cognitive agents and the world.

What attitude should we take towards a theory or model that involves idealizations? A referee suggests that acceptance, as opposed to belief, is a reasonable attitude since we know that the theory or model involves falsehoods but believe that these falsehoods do not affect the intended predictions or explanations. Epistemists, however, would take an alternative attitude, viz., believing that an idealized theory is, although not completely true, approximately true. This epistemic attitude would be endorsed by Stathis Psillos (1999: 276) who claims that the ideal gas law, although not completely true, is approximately true.

\section{Simplicity}

Scientists make progress when they choose a simple theory over a complex one. Dellsén argues that the noetic approach can, while the epistemic approach cannot, account for such progress. This section aims to defend the epistemic approach from this objection.

Suppose that $T_{1}$ and $T_{2}$ are empirically equivalent, but that $T_{1}$ is simple whereas $T_{2}$ is complex, and that simplicity is not an epistemic, but a pragmatic, virtue. The epistemic approach implies that no progress is made when scientists choose $\mathrm{T}_{1}$ over $\mathrm{T}_{2}$, for "the amount of knowledge gained by coming to know $\mathrm{T}_{1}$ is (by any measure) exactly the same as the amount of knowledge gained by coming to know T2" (Dellsén, 2016a: 81). By contrast, the noetic approach implies that progress is made when scientists choose $T_{1}$ over $T_{2}$, for a "simpler theory enables us to explain and predict aspects of the world that would be more difficult or even impossible to explain and predict with a more complex theory" (Dellsén, 2016a: 81). It follows that "the simpler $\mathrm{T}_{1}$ would provide us with more understanding than the more complex $\mathrm{T}_{2}$ " (Dellsén, 2016a: 81).

Dellsén thinks that $T_{1}$ gives rise to more understanding than $T_{2}$, not because he thinks that $T_{1}$ has a broader explanatory and predictive scope than $T_{2}$, but because he thinks that it is psychologically easier to derive observational consequences from $T_{1}$ than from $T_{2}$. For example, while it takes less than a second to derive an observational consequence from $\mathrm{T}_{1}$, it takes more than a year to derive an observational consequence from $\mathrm{T}_{2}$. Given that deriving observational consequences is required to explain and predict phenomena, scientists can explain and predict phenomena more efficiently with $T_{1}$ than with $T_{2}$. It follows that $T_{1}$ contributes more than $T_{2}$ to an increase in understanding, although $T_{1}$ and $T_{2}$ are empirically equivalent. This seems to be what Dellsén has in mind.

Epistemists have a theoretical resource to diffuse this objection. To say that it is psychologically easier to derive observational consequences from $T_{1}$ than $T_{2}$ implies that 
scientists can generate observational knowledge more efficiently with $\mathrm{T}_{1}$ than with $\mathrm{T}_{2}$. It follows that $T_{1}$ contributes more than $T_{2}$ to the accumulation of knowledge, although $T_{1}$ and $\mathrm{T}_{2}$ are empirically equivalent. Therefore, choosing $\mathrm{T}_{1}$ over $\mathrm{T}_{2}$ constitutes scientific progress.

What can we learn from the preceding discussion? The empirical equivalence of $\mathrm{T}_{1}$ and $\mathrm{T}_{2}$ does not mean that $\mathrm{T}_{1}$ and $\mathrm{T}_{2}$ generate observational knowledge at the same rate any more than it means that they generate understanding at the same rate. It is one thing that they are equivalent; it is another that they generate knowledge and understanding with equal efficiency.

\section{Conclusion}

Dellsén argues that understanding requires not belief but acceptance, that scientific understanding arises from correct explanations and predictions, and that scientific progress consists in increasing understanding. I objected that when scientists explain and predict phenomena, they believe at least that the propositions that concern the phenomena are true. If they merely accept the propositions, their peers might suspect that they have fabricated data, and that their explanation, prediction, and understanding do not reflect the world. It follows that understanding, as defined by Dellsén, cannot capture scientific progress.

\section{References}

Bird, Alexander (2007). “What is Scientific Progress?” Noûs 41 (1): 64-89.

Cohen, L. Jonathan (1992). An Essay on Belief and Acceptance. Oxford: Oxford University Press.

Dellsén, Finnur (2016a). "Scientific Progress: Knowledge vs. Understanding”, Studies in History and Philosophy of Science 56: 72-83.

------- (2016b). "Understanding without Justification or Belief”, Ratio. DOI: $10.1111 /$ rati.12134

Einstein, Albert (1905/1956). Investigations on the Theory of the Brownian Movement. Translated by A. D. Cowper. New York: Dover.

Grimm, Stephen (2006). "Is Understanding a Species of Knowledge?", The British Journal for the Philosophy of Science 57 (3): 515-535.

(2014). "Understanding as Knowledge of Causes", In Abrol Fairweather (ed.), Virtue Epistemology Naturalized: Bridges between Virtue Epistemology and Philosophy of Science (Synthese Library, vol. 366). Cham: Springer, pp. 329-345.

Kitcher, Philip (2002). "Scientific Knowledge", In Paul Moser (ed.), The Oxford Handbook of Epistemology. New York: Oxford University Press, pp. 385-408.

Kuhn, Thomas (1962/1970). The Structure of Scientific Revolutions. Chicago: University of Chicago Press.

Laudan, Larry (1977). Progress and Its Problems. London: Routledge and Kegan Paul. 
(1984). Science and Values. Berkeley: University of California Press.

Lipton, Peter (2004). Inference to the Best Explanation. New York: Routledge.

Niiniluoto, Ilkka (1980). Is Science Progressive? Dordrecht: Reidel.

(2014). "Scientific Progress as Increasing Verisimilitude", Studies in History and Philosophy of Science 46: 73-77.

------- (2015). "Optimistic Realism about Scientific Progress", Synthese. DOI: 10.1007/s11229-015-0974-z

Park, Seungbae (2015). “Accepting Our Best Scientific Theories”, Filosofija. Sociologija 26 (3): 218-227.

(2016). "Scientific Realism and Antirealism in Science Education", Coactivity: Philosophy. Communication 24 (1): 72-81.

--------- (2017). “Defense of Epistemic Reciprocalism”, Filosofija. Sociologija. 28 (1): 56-64.

Psillos, Stathis (1999). Scientific Realism: How Science Tracks Truth. New York: Routledge.

Rowbottom, Darrell (2010). "What Scientific Progress Is Not: Against Bird's Epistemic View", International Studies in the Philosophy of Science 24 (3): 241-255.

Saatsi, Juha (2016). "What Is Theoretical Progress of Science?" Synthese. DOI: $10.1007 / \mathrm{s} 11229-016-1118-9$ 